\title{
Estimación del valor financiero de las existencias maderables de cedro rojo (Cedrela odorata L.) en México
}

\section{Estimate of the financial value of timber stocks of red cedar (Cedrela odorata L.) in Mexico}

\author{
José Luis Romo-Lozano"; J. Jesús Vargas-Hernández², Javier López-Upton² y María Luisa Ávila Angulo²
}

1 Universidad Autónoma Chapingo. División de Ciencias Forestales. Texcoco, Estado de México, México.

\author{
2 Colegio de Postgraduados. Posgrado en Ciencias \\ Forestales. Montecillo, Estado de México, México. \\ jjesus.vargashernandez@gmail.com; jlopezupton@ \\ gmail.com; avila.maria@colpos.mx \\ *Autor de correspondencia. jlromo@ayayayale.edu
}

\begin{abstract}
RESUMEN
Uno de los dos grandes rubros de la valoración económica en el ámbito de los servicios ambientales asociados con recursos naturales es la valoración con mercado. Como su nombre lo indica, esta valoración utiliza información que se genera en el proceso de intercambio del bien o servicio valorado. El presente documento contiene un esfuerzo de este tipo de valoración, desde el punto de vista financiero, del cedro rojo (Cedrela odorata L.) en México. En este ejercicio se aplicó el método de precios de mercado para determinar el valor monetario de su principal uso (maderable), considerando el volumen de madera de la especie existente en los bosques naturales, con base en información generada a partir de las bases de datos del inventario nacional forestal. El efecto del volumen de madera, el precio y el costo del transporte sobre el valor total se evaluó mediante un análisis de sensibilidad. El valor maderable estimado de los árboles en pie asciende a la cantidad de MXN\$5 282183404 (USD \$288 328 788, a precios de mayo de 2016). De las tres regiones consideradas en esta valoración, la región Golfo de México es la de mayor aportación. De acuerdo con el análisis de sensibilidad realizado, el precio de la madera es el principal factor que afecta el valor maderable de la especie.
\end{abstract}

PAlabras Clave: bosque natural, especies sujetas a protección especial, maderas preciosas, recursos forestales, valoración con mercado.

\section{ABSTRACT}

One of the two large categories of economic valuation within the environmental services field associated to natural resources is the market valuation. As its name implies, this type of valuation uses information generated in the process of exchange of goods or services valued. This document contains a financial valuation effort of Spanish red cedar (Cedrela odorata L.) species in Mexico. In this exercise the market price method was applied in order to estimate the monetary value of its main use (timber), considering the standing wood volume of the species in natural forests, based on information generated from the national forest inventory database. The effect of wood volume, price and transportation cost on total value was evaluated through a sensitivity analysis. The estimated value for total standing timber was MXN\$ 5282183404 (USD\$288 328788 at May 2016 prices). The Gulf of Mexico region has the highest value representation among the three regions considered in this paper. According to the sensitivity analysis, the price of wood is the main factor affecting total timber value of the species.

KEY WORDS: natural forest, species subjected to special protection, precious hardwoods, forest resources, market valuation.

\section{INTRODUCCIÓN}

El Cedro rojo (Cedrela odorata L.) es una especie de la familia Meliaceae con origen en la América tropical. Su distribución en el territorio de México abarca tanto la vertiente del golfo, desde el sur de Tamaulipas y sureste de San Luis Potosí hasta la península de Yucatán, como la del Pací- fico, desde Sinaloa hasta Guerrero y en la depresión central y la costa de Chiapas. Fuera de México, se distribuye por Centroamérica e islas del Caribe, hasta Brasil; ocurre en climas tropicales y subtropicales húmedos y semihúmedos; cubre desde localidades al nivel del mar en varios países hasta cerca de los 3000 metros en Bolivia (Patiño, 1997). 
Su importancia económica se denota por ser una de las especies maderables que más demanda ha tenido en la industria forestal de México. Su madera, de características excelentes, es usada para obtener madera aserrada y chapa para madera terciada (Morgado, 2014). Entre otros usos, se incluyen la fabricación de muebles finos, instrumentos musicales, productos aromatizantes y medicinales (hoja, raíz, corteza, semilla y tallo) en infusiones para distintos padecimientos (Linnaeus, 1759).

Su importancia ecológica se revela al ser una especie secundaria y primaria a la vez, pues se ha encontrado como especie pionera en la vegetación secundaria de diversas selvas, y también como elemento frecuente en el estrato superior de las selvas maduras. Asimismo, tiene potencial para reforestación en zonas degradadas de selva y zonas secas semiáridas y ha sido utilizada en la rehabilitación de sitios donde hubo explotación minera (Andrade y Solís, 2004).

De acuerdo con la Norma Oficial Mexicana 059 (NOM-059), el cedro rojo en México se encuentra en la categoría "sujeta a protección especial"; es decir, es una especie que podría llegar a encontrarse amenazada por factores que inciden negativamente en la viabilidad de sus poblaciones, por lo que existe la necesidad de promover su recuperación y conservación, junto con las otras especies asociadas en los ecosistemas naturales donde se encuentra.

El cedro rojo, como prácticamente todas las especies forestales, provee servicios ambientales a los cuatro niveles de la biodiversidad: gene, especie, ecosistema y función. En este sentido, la estimación del valor económico de los beneficios que su existencia proporciona presenta dificultades ampliamente reconocidas y abordadas en la perspectiva económica y que se asocian con la imposibilidad de obtener información de mercado (precios) para un amplio vector de tales beneficios (servicios). Son dos las razones por la cuales este tipo de bienes y servicios no genera información de mercado, y consecuentemente les define técnicamente como bienes públicos: la no excluibilidad y la no rivalidad. La primera se refiere al hecho de que una vez que los bienes son producidos presentan muchas dificultades para ser excluidos del uso de los bene- ficiarios, implicando con ello la imposibilidad de imponer cargos (precios), y en consecuencia, imposibilitando o limitando su comercialización. La segunda significa que la agregación de beneficiarios (consumidores) ocurre con un costo marginal cero, lo cual se presenta, en algunos casos, hasta antes de que ocurra algún nivel de congestionamiento en el uso del bien o servicio considerado.

Algunos de los servicios y funciones ecológicas del cedro rojo que caen en la categoría de públicos, y consecuentemente no generan información de mercado para su valoración monetaria, incluyen su papel como depósito de carbono y proveedor de otros servicios ambientales o para mantener la resiliencia del ecosistema y mitigar los impactos del cambio climático, además de su función como fuente de recursos genéticos útiles en ambientes futuros. Por su naturaleza, estos aspectos requieren abordarse por separado de la valoración económica de los bienes de mercado. Por otro lado, los principales beneficios que caen en la categoría de bienes de mercado son la producción de madera y de aceites o substancias de interés farmacéutico y químico; sin embargo, en las condiciones de México, el principal bien de mercado en la actualidad es la madera.

Aun cuando la posibilidad de pérdida total de la especie en el territorio mexicano no se observa como un escenario probable, la estimación de las existencias a valor de mercado responde a la pregunta: ¿qué se pierde si se pierden las existencias del cedro rojo en el país? Se debe señalar, sin embargo, que el valor económico total de lo que se perdería incluye los otros valores de no mercado de la especie, mismos que no se consideran en este estudio.

\section{OBJETIVo}

Debido a su carácter de especie sujeta a protección especial, así como a su importancia ecológica y económica, este trabajo tiene como objetivo estimar el valor financiero de las existencias de cedro rojo en los bosques naturales del país, enfocando la estimación en el valor de uso de la especie en términos de la información de mercado, específicamente, el valor de mercado de la madera en pie en bosques naturales. 


\section{MATERIALES Y MÉTODOS}

La metodología aplicada en esta valoración se desarrolló en tres fases: la primera de ellas fue estimar el número de árboles y volumen de madera en rollo de cedro rojo existente en los bosques naturales del país; la segunda fase se enfocó en la aplicación del método de valoración a precios de mercado, incluyendo un análisis de sensibilidad del valor estimado en respuesta a cambios en algunas de las variables consideradas. Se realizó también durante el mes de mayo de 2015, una fase de campo en la región de la Península de Yucatán con el fin de precisar datos y acopiar información de tipo económico.

\section{Método de estimación de las existencias de cedro rojo}

La realización de esta fase incluyó distintas etapas: la definición de regiones dentro del país donde existe la especie en condiciones naturales, la delimitación del área de distribución natural por tipo de vegetación, y la estimación de parámetros de productividad y estructura de las poblaciones de Cedrela odorata L. en cada región.

\section{División del área de estudio en regiones}

El cedro rojo es una especie abundante y con una amplia distribución en el territorio nacional; crece en distintos suelos, tanto de origen volcánico como calizo, sus máximos tamaños e incrementos se presentan en zonas con precipitación entre $2500 \mathrm{~mm}$ y $4000 \mathrm{~mm}$ anuales (Pennington y Sarukhán, 2005). Aunque se encuentra también en zonas con precipitación menor, el árbol presenta menores incrementos y las poblaciones son más pequeñas. Debido a lo anterior, las principales y posibles áreas de distribución de la especie se agruparon en tres regiones (Fig. 1): a) Golfo de México, que incluye los estados de Tamaulipas, San Luis Potosí, Hidalgo, Querétaro, Puebla, Veracruz, Oaxaca y Tabasco; b) Pacífico, que abarca los estados de Sinaloa, Durango, Nayarit, Jalisco, Colima y Guerrero; y c) Sur-Sureste, que incluye Chiapas, Campeche, Yucatán y Quintana Roo. Es posible encontrar pequeñas poblaciones de la especie en áreas no consideradas en las regiones definidas, sin embargo, éstas han sido excluidas del estudio por considerarse de poca cuantía.

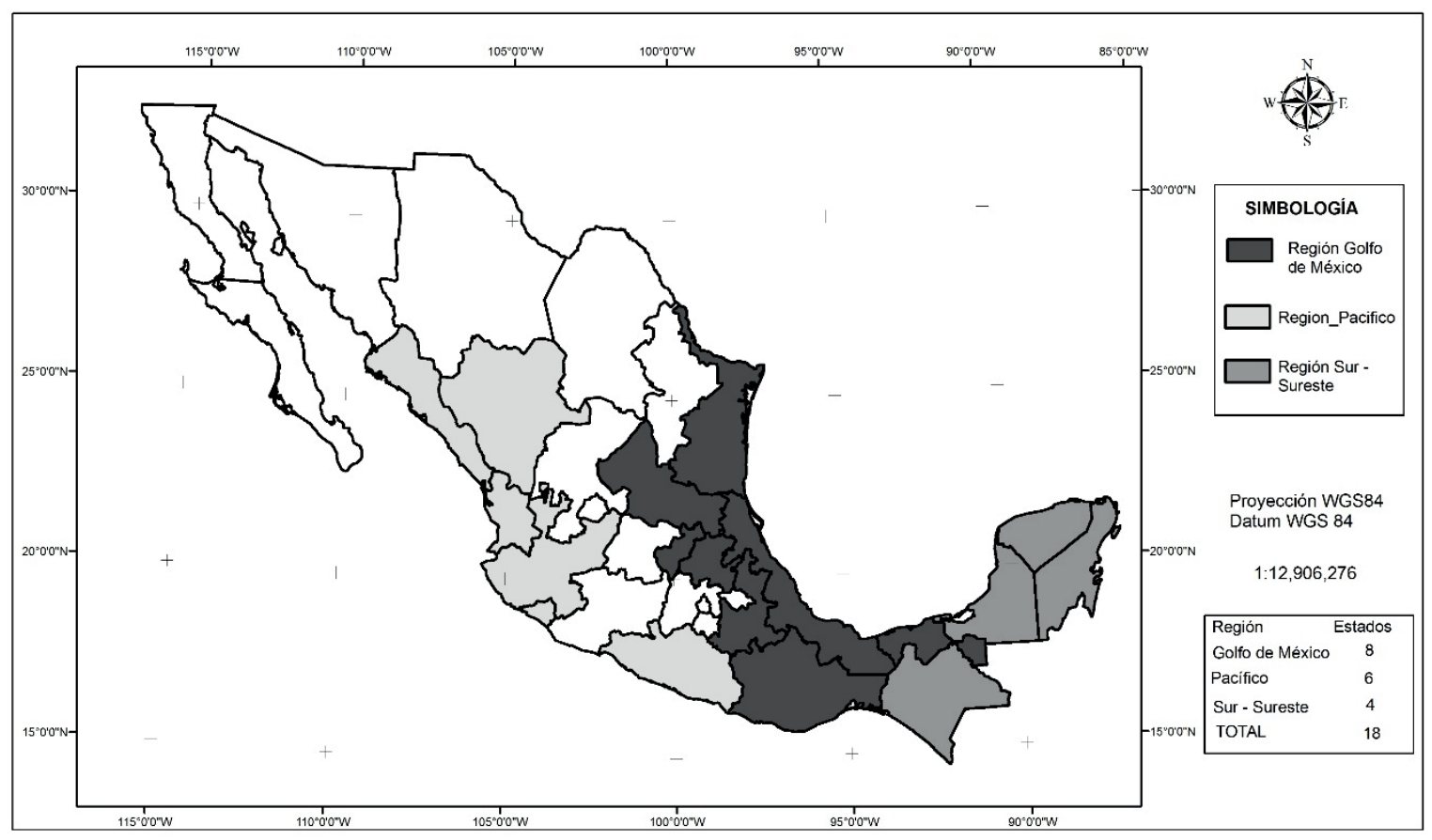

Figura 1. Regionalización del área de distribución de Cedrela odorata L. en México. 
Para la delimitación de las regiones se utilizó el software ArcGis 9.3®, con información digital en formato shapefile sobre los límites estatales obtenidos del marco geoestadístico nacional 2014 del Instituto Nacional de Estadística y Geografía [Inegi] (2014).

\section{Delimitación del área de distribución natural de Cedrela odorata L. por tipo de vegetación}

El área de distribución de Cedrela odorata L. se definió a partir de la información contenida en la carta de Uso de Suelo y Vegetación Serie V de Inegi escala 1:250 000 (Inegi, 2011), los datos de los sitios de muestreo del Inventario Nacional Forestal y de Suelos (INFyS) y los límites de los estados de las regiones Golfo de México, Pacífico y Sur- Sureste en formato digital tipo shapefile. El criterio utilizado fue el tipo de vegetación (selvas altas, selvas medianas y bosque mesófilo) donde se desarrolla preferencialmente la especie.

Mediante el uso del Programa ArcGis 9.3® se ubicaron las regiones y se estimó la superficie por tipo de vegetación, con lo cual se determinó el área total donde se encuentra la especie en forma natural. Este dato se usó en la determinación del número de árboles, área basal total y volumen de madera que se estima en las existencias de cedro rojo por región. La información cartográfica digital utilizó una proyección Cónica Conforme de Lambert, datum WGS 84 y sistema de coordenadas geográficas.

\section{Estimación de parámetros dasométricos y}

estructura de las poblaciones naturales de

\section{Cedrela odorata L. por región}

De la base de datos del INFyS de los años 2009 a 2013, se extrajo la información de Cedrela odorata L. por conglomerado y sitio de muestreo en campo. Las variables consideradas fueron número de árboles, diámetro normal, altura total, altura de fuste limpio, condición del árbol, tipo de vegetación, ubicación geográfica del sitio, estado y municipio. Con estas variables se calculó el número de árboles, el área basal y el volumen de madera por hectárea y por región, utilizando el procedimiento de estimadores de razón (Velasco, Ramírez, Moreno y de la Rosa, 2003).
El área basal por árbol (AB), en metros cuadrados, se estimó con los datos obtenidos del INFyS mediante la ecuación

$$
A B=D N^{2} \times 0.7854
$$

donde DN es el diámetro normal en m. Para calcular el volumen de madera se aplicó la ecuación de volumen de madera en rollo por árbol (V), en metros cúbicos, propuesta por MIQROO con un coeficiente mórfico de 0.6367 , que al ser multiplicado por $\pi / 4$ resulta 0.5

$$
V=0.5 \times D N^{2} \times A F L
$$

donde AFL es la altura de fuste limpio en metros.

Para estimar los valores promedio por hectárea por región, primero se sumaron los valores de número de árboles, $\mathrm{AB}$ y volumen de madera de cedro encontrados en los conglomerados por región, después se determinó el área total de los sitios muestreados para selvas y bosque mesófilo por estado y para cada una de las regiones. Al final se dividió el valor total de cada una de las variables por región entre la superficie total de los sitios muestreados por región.

Las variables número de árboles, área basal y volumen de madera por hectárea se determinaron mediante la estimación de las medias por hactárea, así como la desviación estándar y los límites máximos y mínimos del intervalo de confianza del valor promedio con una confiabilidad de $95 \%$.

Para conocer la estructura de la población de cedro, se elaboraron dos gráficas para cada una de las regiones: la distribución de las clases diamétricas y la distribución de las alturas a partir de los datos del INFyS. En ambas gráficas se contabilizó el número de árboles que estaba comprendido en las distintas categorías diamétricas.

En cada región, por la misma estructura de la población, se tiene distinto número de categorías diamétricas: para la región Golfo de México se definieron 14 categorías, 12 para la región Sur-Sureste, y 11para la región del Pacífico. El intervalo entre categorías diamétricas fue de 5 
cm y el menor tamaño de árbol considerado en las tres regiones fue la categoría de $10 \mathrm{~cm}$.

Con respecto a la distribución de alturas, la región del Golfo de México presentó cuatro categorías y en las regiones del Pacífico y Sur-Sureste fueron tres. La categoría de menor tamaño considerada fue la de $5 \mathrm{~m}$ y la máxima de $30 \mathrm{~m}$.

\section{Fase de campo}

Se realizó una visita de campo en el Municipio de Calakmul, Campeche, en dos ejidos con manejo forestal (20 de Noviembre y Álvaro Obregón), donde se entrevistó a productores y a prestadores de servicios técnicos para recabar datos de crecimiento, producción y precios de madera en rollo de la especie.

\section{Metodología de valoración}

Dentro de la amplia gama de métodos para la valoración económica, existe un conjunto de ellos que utiliza información de mercado. Por el objetivo del presente trabajo, el método aplicado fue el de precios de mercado. Esta metodología utiliza todos los precios de mercado de los bienes y servicios obtenidos del bosque, aunque tiene el inconveniente de no considerar los bienes y servicios sin mercado (Gregersen, Arnold, Lundgren and Contreras, 1995). Los precios de mercado reflejan la disposición a pagar (DAP) por costo y beneficios de las opciones de los terrenos forestales que son comercializados (madera, leña, alimentos medicinas, recreación, etcétera) y tiene la ventaja de que los datos sobre precios son relativamente fáciles de obtener (Knowler y Lovett, 1996). En este estudio lo que procedió fue identificar (estimar) las existencias de madera de cedro en bosques naturales, con base en los datos obtenidos del INFyS, y definir el precio por metro cúbico de madera a pie de brecha, tomando en cuenta algunas consideraciones sobre el transporte para obtener un diferencial en el costo de la madera.

Para el ajuste de precios por costos de transporte al mercado se realizó un análisis mediante el software ArcGis 9.3®, con información digital en formato shapefile de los datos vectoriales de las áreas urbanas consideradas como mercado potencial, obtenidas del marco geoestadístico nacional 2014 del Inegi. Con estos datos se determinaron las áreas de influencia en rangos, desde los $5 \mathrm{~km}$ hasta más de $50 \mathrm{~km}$, y se calcularon la superficie y existencias volumétricas de cedro que se encontraban en los distintos niveles de distancia al mercado.

En el proceso de estimación se considera, en primer lugar, que el aprovechamiento comercial de la madera del cedro rojo en bosques naturales es legal; esto porque es conocida la limitación jurídica que implica el que la especie esté considerada en la categoría "sujeta a protección especial" (Diario Oficial de la Federación, 2010). Además, se presume que el precio de la madera no se afecta por el incremento sustancial en la oferta de madera de la especie y que el precio actual, con base en la información de campo, es de MXN\$3500.00/ $\mathrm{m}^{3}$ a pie de brecha.

\section{Análisis de sensibilidad}

Se realizó un análisis de sensibilidad para observar el efecto de posibles modificaciones en tres variables de interés: volumen, precio y penalización por costos de transporte al mercado potencial. Derivado de ello se construyeron tres escenarios: escenario base, el cual corresponde a las estimaciones de volumen promedio realizadas inicialmente; escenario -20, el cual consistió en disminuir en $20 \%$ las tres variables de interés; y el escenario +20 en el que de manera similar se aumentaron en $20 \%$ las mismas variables. Posteriormente, para cada modificación se mantuvo sin cambio el resto de las variables consideradas en el escenario base.

\section{RESULTADOS Y DISCUSIÓN}

\section{Estimación de existencias volumétricas de cedro rojo}

La distribución de Cedrela odorata L., determinada en las tres grandes regiones, se muestra en la figura 2. La superficie está relacionada con el tipo de vegetación de selvas altas, selvas medianas y bosque mesófilo, que son los principales tipos de vegetación en los que se reconoce la presencia de la especie en la base de datos del INFyS. 
Esta estimación es un tanto conservadora, puesto que también se ha mencionado la presencia de la especie en otros tipos de vegetación, ya sea como parte de la vegetación secundaria o por la existencia de ecotonos y traslapes entre diferentes asociaciones vegetales. Aun así, se consideró preferible utilizar este enfoque conservador y subestimar las existencias reales de la especie que incurrir en el error opuesto al incluir en la estimación otros tipos de vegetación. En la región Sur-Sureste (Península de Yucatán) se presenta la mayor superficie con 2.26 millones de hectáreas con presencia de cedro rojo; la región Golfo presenta 1.3 millones de hectáreas de selvas y bosque mesófilo con cedro rojo, mientras que la región con menor superficie de tipos de vegetación con presencia de cedro rojo es la denominada Pacífico.

En la estimación del número de árboles de cedro rojo por hectárea, resultó que los valores promedio más altos se encuentran en la región Golfo de México. Del mismo modo, en esta misma región, se observa la mayor área basal por unidad de superficie, con valor de $0.442 \mathrm{~m}^{2} / \mathrm{ha}$ y el máximo volumen de $1.39 \mathrm{~m}^{3} / \mathrm{ha}$ en promedio. Los menores valores se presentaron en la región Sur-Sureste con un volumen promedio de $0.273 \mathrm{~m}^{3} /$ ha (Tabla 1 ).

La tabla 2 muestra los valores totales estimados para cada región del área de distribución natural. En la región Golfo de México se estima la presencia de 9.06 millones de árboles de un total de 14.98 millones en las tres regiones; igualmente, esta región tiene la mayor existencia de volumen de madera con 1.8 millones de $\mathrm{m}^{3}$. La región Pacífico es la que tiene menor existencia de árboles con 1.4 millones en una superficie de 556 mil hectáreas. La región con mayor superficie de vegetación con existencias de cedro rojo es la Sur-Sureste con 2.2 millones de hectáreas, pero debido a la menor densidad y tamaño promedio de los árboles, el número total de árboles y las existencias volumétricas son menores que en la región Golfo de México.

En las tres regiones geográficas el mayor número de árboles tiene diámetros entre $10 \mathrm{~cm}$ y $20 \mathrm{~cm}$, lo cual indica que predominan los árboles jóvenes; solamente en la región Golfo hay presencia de algunos árboles maduros y sobre maduros. En la región Sur-Sureste solo 1\% de los

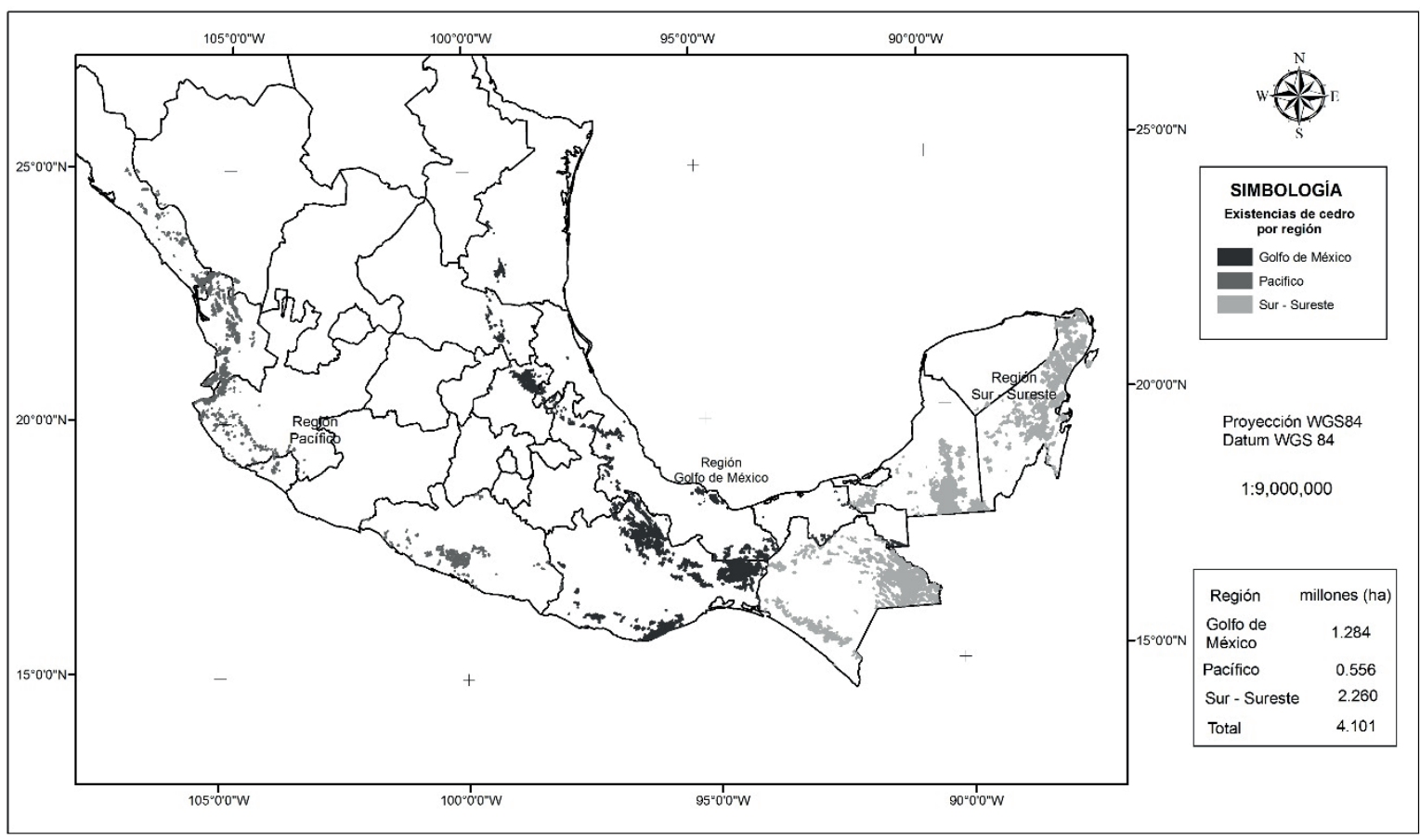

Figura 2. Distribución de las existencias actuales de Cedrela odorata L. de acuerdo con la base de datos del INFyS 2009-2013. 
Tabla 1. Valores promedio de número de árboles, área basal y volumen de madera por hectárea en las tres regiones del área de distribución natural de Cedrela odorata L.

\begin{tabular}{ccccccccccc}
\hline \multirow{2}{*}{ Región } & \multicolumn{3}{c}{$\begin{array}{c}\text { Densidad } \\
\text { (No. árboles/ha) }\end{array}$} & \multicolumn{3}{c}{$\begin{array}{c}\text { Área basal } \\
\left(\mathrm{m}^{2} / \mathrm{ha}\right)\end{array}$} & \multicolumn{3}{c}{$\begin{array}{c}\text { Volumen madera } \\
\left(\mathrm{m}^{3} / \mathrm{ha}\right)\end{array}$} \\
\cline { 2 - 11 } & Media & Lím. inf. & Lím. sup. & Media & Lím. inf. & Lím. sup. & Media & Lím. inf. & Lím. sup. \\
\hline Pacífico & 2.51 & 0.84 & 4.18 & 0.12 & 0.05 & 0.19 & 0.34 & 0.12 & 0.56 \\
Sur-Sureste & 2.00 & 1.23 & 2.78 & 0.06 & 0.04 & 0.08 & 0.27 & 0.18 & 0.37 \\
Golfo de México & 7.05 & 4.83 & 9.27 & 0.44 & 0.27 & 0.61 & 1.39 & 0.88 & 1.9 \\
\hline
\end{tabular}

TAвla 2. Valores promedio estimados de número de árboles, área basal y volumen de madera totales en las tres regiones del área de distribución natural de Cedrela odorata L.

\begin{tabular}{ccccc}
\hline Región & Superficie (ha) & $\begin{array}{c}\text { No. de árboles } \\
\text { (millones) }\end{array}$ & $\begin{array}{c}\text { Área basal } \\
(\text { miles m })^{2}\end{array}$ & $\begin{array}{c}\text { Volumen madera } \\
(\text { miles m }\end{array}$ \\
\hline Pacífico & 556834 & $1.397 \pm 0.927$ & $65.71 \pm 38.98$ & $188.77 \pm 124.73$ \\
Sur-Sureste & 2260173 & $4.524 \pm 1.747$ & $135.61 \pm 40.68$ & $617.03 \pm 214.72$ \\
Golfo de México & 1284139 & $9.057 \pm 2.849$ & $567.59 \pm 220.87$ & $1784.95 \pm 653.63$ \\
Total & 4101146 & 14.978 & 768.91 & 2590.75 \\
\hline
\end{tabular}

individuos muestreados por el INFyS tiene diámetro mayor o igual a $50 \mathrm{~cm}$, por lo que en la región Golfo de México la estructura poblacional refleja una condición más adulta y con mayores dimensiones en altura y diámetro que en las otras regiones.

\section{Estimación del valor de mercado de la madera}

Dado que la distribución de la madera se encuentra a distintas distancias de los centros de posible colocación, se construyó una tabla donde, a partir de la información geográfica disponible, se determinaron las áreas y volumen correspondiente en relación con la distancia a los centros urbanos.

Adicionalmente, se consideraron los costos en que se incurre antes de que el volumen de madera llegue a pie de brecha, los cuales incluyen: planeación, derribo y traslado a pie de brecha, entre otros. Estos registran un monto total entre MXN\$1430/ha y MXN\$1500/ha. Sin embargo, este monto se distribuye entre el grupo de especies aprovechadas en cada hectárea de superficie, por lo que se considera que al cedro solamente le corresponde 1/3 del punto medio de estos costos, es decir MXN\$488.33.

Posteriormente se estableció una penalización en el precio de MXN $\$ 20 / \mathrm{km} / \mathrm{m}^{3}$, debido a costos de transporte de la madera al mercado, tomando el punto medio de cada intervalo de distancia (información de campo y consenso de equipo experto). En la tabla 3 se muestra el valor de la madera, considerando los costos incurridos hasta su traslado a pie de brecha y la penalización por distancia a los centros de mercado. De acuerdo con estos resultados, el valor total de la madera de cedro rojo existente en rodales naturales se estima en más de cinco mil millones de pesos; dos terceras partes del valor maderable se concentra en la región del Golfo de México, con más de 3.59 mil millones de pesos; la región Sur-Sureste representa $23.5 \%$ del valor total y el resto se encuentra en la región Pacífico. 
Tabla 3. Valor de la madera por región, con precio penalizado por distancia a centros urbanos.

\begin{tabular}{|c|c|c|c|c|c|}
\hline Distancia (km) & Precio (\$) & Unidades & Región Sur-Sureste & Región Pacífico & Región Golfo de México \\
\hline \multirow[t]{2}{*}{$0-5$} & 2966.67 & Volumen $\left(\mathrm{m}^{3}\right)$ & 4043 & 3004 & 17867 \\
\hline & & Valor/región (\$) & 11995212 & 8911260 & 53006001 \\
\hline \multirow[t]{2}{*}{$5-10$} & 2866.67 & Volumen $\left(\mathrm{m}^{3}\right)$ & 19810 & 14407 & 62964 \\
\hline & & Valor/región (\$) & 56787705 & 41301451 & 180497791 \\
\hline \multirow[t]{2}{*}{$10-20$} & 2716.67 & Volumen $\left(\mathrm{m}^{3}\right)$ & 99151 & 48568 & 247587 \\
\hline & & Valor/región (\$) & 269359733 & 131942412 & 672613405 \\
\hline \multirow[t]{2}{*}{$20-30$} & 2516.67 & Volumen $\left(\mathrm{m}^{3}\right)$ & 105370 & 49309 & 346328 \\
\hline & & Valor/región (\$) & 265181739 & 124093782 & 871592748 \\
\hline \multirow[t]{2}{*}{$30-40$} & 2316.67 & Volumen $\left(\mathrm{m}^{3}\right)$ & 103108 & 30745 & 257981 \\
\hline & & Valor/región (\$) & 238866082 & 71226413 & 597655782 \\
\hline \multirow[t]{2}{*}{$40-50$} & 2116.67 & Volumen $\left(\mathrm{m}^{3}\right)$ & 98362 & 27437 & 316897 \\
\hline & & Valor/región (\$) & 208200818 & 58076001 & 670766937 \\
\hline \multirow[t]{2}{*}{$>50$} & 1016.67 & Volumen $\left(\mathrm{m}^{3}\right)$ & 187184 & 15296 & 535329 \\
\hline & & Valor/región (\$) & 190303932 & 15551478 & 544252722 \\
\hline Total & & Valor/región (\$) & 1240695221 & 451102797 & 3590385386 \\
\hline
\end{tabular}

\section{Análisis de Sensibilidad}

En el análisis de sensibilidad se estimó el efecto de las variables consideradas en el valor del volumen de la madera por región y en su agregación total (Tabla 4). Los resultados expresan que la elasticidad del valor del volumen con respecto a los cambios en las variables de interés es mayor a 1 en el caso del precio; igual a 1 para la variable volumen de madera; y menor a 1 para la variable penalización (Fig. 3). Esto es así porque ante una disminución o incremento de $20 \%$ en el precio, el valor del volumen de la madera se disminuye o incrementa en una proporción mayor, 34\%. Para el caso de la penalización ocurre que al aumentarla o disminuirla en $20 \%$, el valor del volumen de la madera se modifica en un una proporción menor, 9.6\%. La proporción de cambio en la variable volumen de madera causa un cambio en la misma proporción en el valor del volumen de madera.

El hecho de que la elasticidad del valor del volumen de la madera, ante cambios en el precio, sea mayor que 1 , y esa misma elasticidad con respecto a los cambios en la penalización sea menor que 1 , se explica a partir de que el factor multiplicativo que convierte el volumen de madera en valor tiene a ambos componentes: precio y penalización, esto es

$$
\text { ValMad=(Pr-Pn })(\text { VolMad })
$$

Donde:

Valmad: Valor de la madera

Volmad: Volumen de madera

Pr: $\quad$ precio

$P n: \quad$ penalización

Asimismo, se observa que en los datos utilizados el monto del precio es demasiado grande en relación con el monto de la penalización, lo que causa que, ante las modificaciones en proporciones iguales de ambas variables, sus efectos sean proporciones significativamente diferentes. En el caso de los cambios en la variable volumen de la madera, el efecto es directo. 
Tabla 4. Sensibilidad del valor del volumen de la madera ante cambios en las variables volumen de madera, precio y penalización por costos de transporte al mercado.

\begin{tabular}{ccccc}
\hline Variable y escenario & \multicolumn{4}{c}{ Valor en millones de pesos mexicanos } \\
\cline { 2 - 5 } & $\begin{array}{c}\text { Región Península } \\
\text { de Yucatán }\end{array}$ & Región Pacífico & Región Golfo & Total \\
\hline Volumen: & 992.56 & 360.88 & 2872.31 & 4225.75 \\
\hline-20 & 1240.70 & 451.10 & 3590.39 & 5282.18 \\
Base & 1488.83 & 541.32 & 4308.46 & 6338.62 \\
+20 & 808.78 & & 2340.92 & 3468.66 \\
\hline Precio: & 1240.70 & 318.97 & 3590.39 & 5282.18 \\
\hline -20 & 1672.61 & 451.10 & 4839.85 & 7095.71 \\
\hline Base & & 583.24 & & \\
+20 & 1364.83 & 474.77 & 3949.23 & 5788.83 \\
\hline Penalización: & 1240.70 & 451.10 & 3590.39 & 5282.18 \\
\hline -20 & 1116.56 & 427.43 & 3231.54 & 4775.53 \\
\hline Base & & & & \\
\hline
\end{tabular}

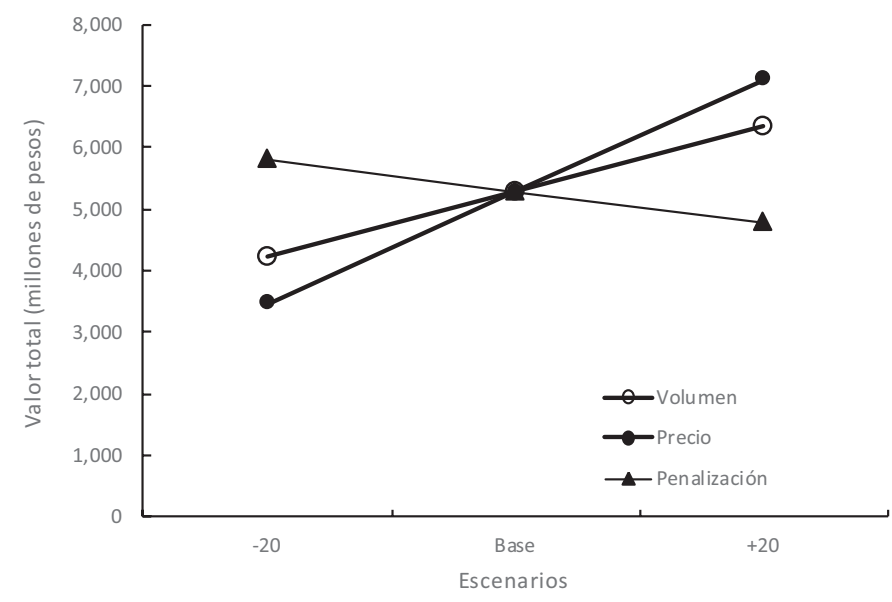

Figura 3. Análisis de sensibilidad del valor financiero del volumen de madera de cedro rojo en tres escenarios de volumen de madera, precio y penalización por costos de transporte.

\section{CONCLUSIONES}

La estimación del valor total de la madera en pie de cedro rojo en México, en el marco de condiciones y restricciones señaladas, es de MXN\$ 5282183403 pesos. La mayor cantidad de existencia maderable en el país se encuentra en la Región Golfo de México, con un valor estimado de MXN\$3 590385 385; le sigue la Región Sur-Sureste (MXN\$1 240695 221) y Región Pacífico (MXN\$451 102 796).

De los beneficios de mercado más conocidos, uso maderable y de aceites químicos y farmacéuticos, el menos desarrollado es el segundo, razón por la cual existe muy poca información sobre el mismo.

De las variables consideradas en el análisis, el precio de la madera es la que muestra una mayor sensibilidad ya que una variación de $20 \%$ ocasiona una variación de $34 \%$ en el valor de la madera.

\section{REFERENCIAS}

Andrade, T. A. y Solís, R. L. Y. (2004). Las bondades del cedro rojo. La Ciencia y El Hombre, 17(3), 17-19.

Diario Oficial de la Federación. (2010). Norma Oficial Mexicana NOM-059-2010, Protección ambiental-Especies nativas de México de flora y fauna silvestres-Categorías de riesgo y especificaciones para su inclusión, exclusión o 
cambio-Lista de especies en riesgo. Recuperado de http:// www.profepa.gob.mx/innovaportal/file/435/1/

NOM_059_SEMARNAT_2010.pdf

Gregersen, H. M., Arnold, J. E. M., Lundgren, A. L. y Contreras, H. A. (1995). Valuing forests: context, issues and guidelines. Rome: FAO

Instituto Nacional de Estadística y Geografía. (2011). Conjunto de datos vectoriales de uso del suelo y vegetación. Escala 1:250 000, Serie V. DGG-INEGI, México. Recuperado de http://www.inegi.org.mx/geo/contenidos/recnat/usosuelo/.

Instituto Nacional de Estadística y Geografía. (2014). Áreas geoestadísticas estatales conjunto de datos vectoriales del marco geoestadístico. Escala 1:1 000 000. DGGINEGI, México. Recuperado de http://www.inegi.org. $\mathrm{mx} /$ geo/contenidos/geoestadistica/m_geoestadistico_2014.aspx

Knowler, D. y Lovett, J. (1996). Training for Environmental Assessment in forestry. Rome: FAO.

Linnaeus, C. (1759). Regnum Vegetabile (Vol. 2). En Systema Naturae (10a ed.) Stockholm: Laurentius Salvius.
Morgado V. S. (2014). Estimación del Rendimiento de Cedro Rojo (Cedrela odorata L.) en el Sureste de México. Tesis de Licenciatura no publicada. Universidad Autónoma Chapingo, Chapingo, Estado de México.

Patiño V. F. (1997). Recursos genéticos de Swietenia y Cedrela en los neotrópicos: Propuesta para Acciones Coordinadas. Recuperado de http://www.fao.org/docrep/006/ AD111S/AD111S00.HTM

Pennington, T. D., y Sarukhán, J. (2005). Árboles Tropicales de México ( $3^{\mathrm{a}}$ ed.). Ciudad de México, México: UNAM y FCE.

Velasco, B. E., Ramírez, M. H., Moreno, S. F. y de la Rosa, V. A. (2003). Estimadores de razón para el inventario nacional forestal de México. Revista Ciencia Forestal en México, 28(94), 23-44.

Manuscrito recibido el 5 de mayo de 2016

Aceptado el 24 de enero de 2017.

Este documento se debe citar como:

Romo-Lozano, J. L., Vargas-Hernández, J. J., López-Upton, J. y Ávila Angulo, M. L. (2017) Estimación del valor financiero de las existencias maderables de cedro rojo (Cedrela odorata L.) en México. Madera y Bosques, 23(1), 111-120. doi: 10.21829/myb.2017.231473 